\title{
FATURAMENTO DE CURATIVOS GRAU II E REGISTROS: CONTRIBUIÇÕES DA ENFERMAGEM ${ }^{1}$
}

\author{
Lucimeire Fermino Lemos², Maria Alves Barbosa³, Alessandra Rodrigues de Almeida Lima ${ }^{4}$, Regiane Aparecida \\ Santos Soares Barreto ${ }^{5}$, Karina Suzuki ${ }^{6}$, Marinésia Aparecida do Prado
}

${ }^{1}$ Texto extraído da tese - Análise dos registros de curativos em prontuários de um hospital de ensino no Estado de Goiás, apresentada ao Programa de Pós-Graduação em Enfermagem da Universidade Federal de Goiás (UFG), em 2016.

${ }^{2}$ Doutora em Enfermagem. Professora da Faculdade de Enfermagem da UFG. Goiânia, Goiás, Brasil. E-mail: luciscats@gmail.com

${ }^{3}$ Doutora em Enfermagem Professora da Faculdade de Enfermagem da UFG. Goiânia, Goiás, Brasil. E-mail: maria.malves@ gmail.com

${ }^{4}$ Doutora em Ciências da Saúde. Auditora na Secretaria Estadual de Saúde de Goiás. Goiânia, Goiás, Brasil. E-mail: aleepm@ hotmail.com

${ }^{5}$ Doutora em Ciências da Saúde. Professora da Faculdade de Enfermagem da UFG. Goiânia, Goiás, Brasil. E-mail: remajuau@ yahoo.com.br

${ }^{6}$ Doutora em Enfermagem na Saúde do Adulto Professora da Faculdade de Enfermagem da UFG. Goiânia, Goiás, Brasil. E-mail: karina.fen@gmail.com

${ }^{7}$ Doutora em Enfermagem. Professora da Faculdade de Enfermagem da UFG. Goiânia, Goiás, Brasil. E-mail: marinesiaprado@ gmail.com

\section{RESUMO}

Objetivo: este estudo verificou se uma ação educativa desenvolvida com enfermeiros contribuiu para o registro de curativos e consequentemente para o aumento do faturamento do procedimento curativo grau II.

Método: estudo exploratório, descritivo quantitativo. Os dados foram coletados das folhas de prescrição e evolução do prontuário e no Centro de Processamento de dados do hospital, antes e após ação educativa, cujo enfoque foi a importância dos registros de enfermagem. Obteve-se 532 ocorrências, sendo 266 antes e 266 após a intervenção. As variáveis foram analisadas segundo suas frequências e porcentagens, a saber: prescrição, evolução, aprazamento, checagem do curativo, categoria profissional do prescritor e do executor do procedimento. Para análise de associações entre variáveis, utilizou-se os testes Qui-quadrado ou Teste de Diferença de Proporção.

Resultados: após a ação educativa, verificou-se o aumento no número dos registros de prescrições, aprazamentos e checagens, fato que interfere diretamente no faturamento deste procedimento, cujo demonstrativo apresentado pela unidade correspondeu a $R \$ 9.201,60$ antes e R\$ 25.142,40 após a ação. Não houve alteração significativa no registro da evolução do procedimento.

Conclusão: desta forma, evidenciou-se o aumento das prescrições após a ação educativa, o que por sua vez, resultou em aumento no faturamento. Assim, recomenda-se a proposição de estudos referentes ao faturamento, relacionado aos registros das ações efetuadas pela enfermagem, assim como o investimento dos gestores em ações educativas.

DESCRITORES: Faturamento. Prescrição. Registros. Curativo. Enfermagem.

\section{BILLING OF LEVEL II WOUND TREATMENT AND RECORDS: NURSING CONTRIBUTIONS}

\begin{abstract}
Objective: to verify whether an educational action developed with nurses contributed to improved documentation on wound treatment, thereby enabling more accurate billing related to level II wound treatment

Methods: exploratory, descriptive and quantitative study. Data were collected from the prescription and evaluation sheets of the clinical record, and from the hospital data processing center, before and after the educational action focusing on the importance of nursing records. Among 532 events reviewed, 266 were before and 266 after the intervention. The variables were analyzed according to frequencies and percentages, namely: prescription, evaluation, schedule time, documentation, professional category of the prescriber and the person performing the procedure. The associations between variables were analyzed using the Chi-square or the test for the difference in proportions.

Results: the amount of prescriptions, schedule time, and documentation increased after the educational action, which directly influences billing for the procedure; costs were $\mathrm{R} \$ 9,201.60$ before and $\mathrm{R} \$ 25,142.40$ after the action. No significant change was identified in the nursing evaluation related to the procedure.

Conclusion: an increase in prescriptions after the educational action was verified, which, in turn, resulted in an increase in billable services. Further studies involving billing as a result of nursing records of interventions are necessary, as well as the investment of the managers in educational actions.
\end{abstract}

DESCRIPTORS: Billing. Prescriptions. Nursing records. Wound healing. Nursing. 


\section{FACTURACIÓN DE CURATIVOS DE GRADO II Y REGISTROS: CONTRIBUCIONES DE LA ENFERMERÍA}

\section{RESUMEN}

Objetivo: este estudio verificó si una acción educativa desarrollada con enfermeros contribuyó para el registro de curativos y, consecuentemente, para el aumento de la facturación del procedimiento curativo del grado II.

Método: estudio exploratorio y descriptivo-cuantitativo. Los datos fueron obtenidos de las hojas de prescripción y evolución del prontuario y en el Centro de Procesamiento de datos del hospital, antes y después de una acción educativa, cuyo enfoque fue la importancia de los registros de enfermería. Se obtuvieron 532 ocurrencias, siendo 266 antes y 266 después de la intervención. Las variables fueron analizadas según sus frecuencias y porcentajes, a saber: prescripción, evolución, aplazamiento, chequeo del curativo, categoría profesional del prescriptor y del ejecutor del procedimiento. Para el análisis de asociaciones entre variables se utilizaron los testes Chi-cuadrado o el Test de Diferencia de Proporción.

Resultados: después de la acción educativa, se verificó el aumento en el número de los registros de prescripciones, aplazamientos y chequeos, hecho que interfiere directamente en la facturación de este procedimiento y cuyo demostrativo presentado por la unidad correspondió a $\mathrm{R} \$ 9.201,60$ antes y $\mathrm{R} \$ 25.142,40$ después de la acción. No hubo alteración significativa en el registro de la evolución del procedimiento.

Conclusión: de esta forma, se evidenció el aumento de las prescripciones después de la acción educativa, y así, resultó en el aumento de la facturación. Por lo tanto, se recomienda la proposición de estudios referentes a la facturación, relacionados con los registros de las acciones efectuadas por la enfermería, así como la inversión de los gestores en acciones educativas.

DESCRIPTORES: Facturación. Prescripción. Registros. Curativo. Enfermería.

\section{INTRODUÇÃO}

Importante para todas as áreas do conhecimento, o registro está presente em nosso cotidiano, e na área da saúde é útil, tanto para o acompanhamento clínico dos pacientes, quanto para a avaliação da assistência prestada, sendo fundamental para o faturamento hospitalar. ${ }^{1}$ Em uma época em que a preocupação com custos se faz presente na administração de serviços de saúde, a falta de registro ou o registro inadequado que leve a perdas no faturamento hospitalar é fator de grande relevância. ${ }^{2}$

Faturamento é o "conjunto dos recebimentos expressos em unidades monetárias, obtidos por uma empresa com a venda de bens ou serviços em determinado período", ${ }^{3}$ portanto, o faturamento hospitalar é o recebimento de repasse correspondente ao processamento dos dados referentes à assistência prestada. ${ }^{4}$

O pagamento por meio do Sistema Único de Saúde (SUS) obedece a um teto financeiro acordado por meio de contratualização entre as unidades prestadoras de serviço e gestor municipal ou estadual de saúde. ${ }^{5}$ Toda internação hospitalar gera a cobrança de um procedimento clínico ou cirúrgico, previsto em tabela, que deverá estar de acordo com o procedimento clínico ou cirúrgico que motivou a internação. Pelo fato do pagamento pela assistência de enfermagem, encontrar-se incluído nas diárias hospitalares ou em taxas de procedimentos, ${ }^{6}$ não se consegue determinar com precisão a contribuição da enfermagem no faturamento em instituições públicas.

O procedimento curativo grau II, sendo classificado como procedimento especial, possui uma peculiaridade em seu faturamento, podendo ser pago em uma Autorização de Internação Hospitalar (AIH) principal, agregando valor a esta. Este fato possibilita a verificação do que foi faturado pela prescrição do procedimento, que é o tratamento de lesão aberta, na qual há grande área de tecido afetado. Desta forma, seu faturamento está condicionado ao registro, ou seja, à prescrição efetuada por médico ou enfermeiro. Existe um limite máximo de 31 procedimentos por mês, por paciente. A cada prescrição deste procedimento, é agregado o valor de R $\$ 32,40$ (trinta e dois reais e quarenta centavos) na fatura hospitalar. ${ }^{7}$

O adequado registro da prescrição, checagem, aprazamento, evolução e da execução do procedimento, poderá auxiliar na minimização de perdas decorrentes do subfaturamento, contribuir para a visibilidade da enfermagem e evidenciar a importância dos registros para os profissionais da saúde. Desta forma, pretendeu-se verificar se uma ação educativa contribuiria no registro de curativos e consequentemente no aumento do faturamento do procedimento curativo grau II.

\section{MÉTODO}

Trata-se de estudo exploratório, descritivo, realizado em uma instituição hospitalar de ensino da Região Centro Oeste do Brasil.

Foi desenvolvida ação educativa, em maio de 2015, com a finalidade de ressaltar a importância dos registros de enfermagem de forma geral e especificamente em relação ao Curativo Grau II. A atividade educativa teve como público alvo os enfermeiros da instituição, com 08 horas/aula desenvolvidas em dois módulos. O primeiro contextualizou os registros e sua relevância para a ocorrência de auditoria. 
No segundo, foram abordadas questões referentes ao modelo de faturamento do SUS, registros de Curativo Grau II e o condicionamento da prescrição e checagem deste procedimento para o devido faturamento hospitalar.

O método de ensino-aprendizagem utilizado foi apresentação expositiva dialogada, possibilitando momentos de debate, fomentando a discussão entre os enfermeiros. A atividade foi desenvolvida duas vezes, uma vez no período matutino e outra no vespertino, de forma a favorecer a participação de todos os enfermeiros. Para avaliar a efetividade da ação educativa, os dados foram coletados em prontuários antes e após e comparados com o faturamento hospitalar do ano em que ação foi realizada.

A coleta dos dados ocorreu a partir de duas fontes - as folhas de prescrição e evolução dos prontuários - em dois momentos distintos: em fevereiro (coleta anterior à ação educativa) e julho de 2015 (coleta posterior à ação educativa). Foram incluídos pacientes internados nas clínicas médica, cirúrgica e ortopédica, maiores de 18 anos e que apresentassem demanda por curativo grau II. As informações do faturamento dos curativos, referentes ao ano de 2015 foram obtidas junto ao Centro de Processamento de Dados (CPD) do hospital.

Os dados extraídos dos prontuários foram informações quanto às prescrições, aprazamentos, checagens, execuções e evoluções do procedimento por profissionais envolvidos na assistência (enfermeiros, médicos, técnicos de enfermagem, residentes e acadêmicos de medicina e enfermagem).

Para a coleta de tais informações, foi utilizado um check-list validado por experts, aplicado antes e após ação educativa. A amostragem de prontuários de fevereiro de 2015 encerrou-se em 266 ocorrências do procedimento em questão, com todas as demandas dos pacientes pelo curativo grau II. Em julho de 2015, utilizou-se nova amostragem, esta, por conveniência para atingir o mesmo número de ocorrências, a partir da demanda por curativo, seguindo-se os mesmos critérios.

Para análise estatística, utilizou-se a ferramenta SPSS/IBM - Statistical Package for Social Science, versão 21.0. Foram analisadas as variáveis: prescrição, aprazamento, checagem do curativo, prescritor e executor do procedimento. As variáveis qualitativas foram apresentadas segundo frequências e porcentagens e suas associações pelo teste Qui-quadrado ou o Teste de Diferença de Proporção.

O projeto, de CAAE 26740514.0.0000.5078, foi aprovado pelo Comitê de Ética do Hospital das Clínicas - UFG, sob o número 616.998/14.

\section{RESULTADOS}

De acordo com a tabela 1, após a intervenção educativa ocorreram 219 (82,3\%) prescrições, 197 (74\%), aprazamentos e $190(71,4 \%)$ checagens de curativos. Assim, a intervenção educativa mostrouse eficaz uma vez que esses registros apresentaram resultados estatisticamente significativos $(p<0,001)$.

Observou-se também mudança na frequência das evoluções $(p=0,018)$, em ambos os momentos.

Tabela 1 - Registros de curativo grau II, antes $(n=266)$ e após (n=266). Goiânia-GO, Brasil, 2015. $n=266$

\begin{tabular}{lccc}
\hline \multirow{2}{*}{ Prescrição } & \multicolumn{2}{c}{ Momento } & p \\
\cline { 2 - 3 } & Antes n(\%) & Depois n(\%) & \\
\hline Sim & $133(50)$ & $219(82,3)$ & $<0,001$ \\
Não & $133(50)$ & $47(17,7)$ & \\
Aprazamento & & & \\
Sim & $68(25,6)$ & $197(74)$ & $<0,001$ \\
Não & $198(74,4)$ & $69(26)$ & \\
Checagem & & & \\
Sim & $67(25,2)$ & $190(71,4)$ & $<0,001$ \\
Não & $199(74,8)$ & $76(28,6)$ & \\
Evolução & & & \\
Sim & $70(26,3)$ & $89(35,5)$ & 0,018 \\
Não & $196(73,7)$ & $177(66,5)$ & \\
Total & 266 & 266 & \\
\hline
\end{tabular}

Teste: Diferença de Proporção

Dentre os casos onde houve o correto registro da prescrição, verificou-se que aquelas efetuadas por enfermeiros antes e após a ação educativa elevaram-se em $51,6 \%$, o que influenciou na diminuição proporcional das prescrições de 38,8\% $(p<0,001)$ entre os médicos, e de $10 \%(p<0,001)$ entre os acadêmicos (Tabela 2).

Tabela 2 - Prescrição de curativo grau II, antes $(n=133)$ e após $(n=219)$ ação educativa, segundo categoria profissional. Goiânia-GO, Brasil, 2015.

\begin{tabular}{lccc}
\hline Profissional & \multicolumn{2}{c}{ Momento } & p \\
\cline { 2 - 3 } & Antes n(\%) & Depois n(\%) & \\
\cline { 2 - 3 } Médico & $76(57,1)$ & $40(18,3)$ & $<0,001$ \\
Enfermeiro & $37(27,8)$ & $174(79,4)$ & $<0,001$ \\
Acadêmico & $14(10,5)$ & $1(0,5)$ & $<0,001$ \\
Residente & $1(0,8)$ & $3(1,3)$ & 0,142 \\
Sem identifi- & $5(3,8)$ & $1(0.5)$ & 0,013 \\
cação & & & \\
Total & $133(100)$ & $219(100)$ & \\
\hline
\end{tabular}

Teste: Diferença de Proporção. 
Em relação às execuções do procedimento, levando-se em conta as ocorrências com o devido registro da execução de curativos, observou-se melhoria, após a ação educativa, quanto à identificação do profissional executor dos procedimentos, passando de $159(61 \%)$ para apenas $27(10,4 \%)$, os registros de curativos sem assinatura profissional $(p<0,001)$ (Tabela 3). Não houve diferença no número de ocorrências onde o curativo não foi executado ou não houve o devido registro da execução ( $n=5$ antes e após a ação educativa).

Tabela 3 - Execução do curativo grau II, antes $(n=261)$ e após $(n=261)$ ação educativa, segundo categoria profissional. Goiânia-Go, Brasil, 2015.

\begin{tabular}{lccc}
\hline Profissional & \multicolumn{2}{c}{ Momento } & p \\
\cline { 2 - 3 } & Antes n(\%) & Depois n(\%) & \\
\hline Médico & $3(1,1)$ & $10(3,8)$ & 0,012 \\
Enfermeiro & $29(11,1)$ & $59(22,6)$ & $<0,001$ \\
Acadêmico & $56(21,4)$ & $34(13)$ & 0,003 \\
Técnico & $14(5,4)$ & $121(46,4)$ & $<0,001$ \\
Residente & $0(0)$ & $10(3,8)$ & $<0,001$ \\
Sem identificação & $159(61)$ & $27(10,4)$ & $<0,001$ \\
Total & $261(100)$ & $261(100)$ & \\
\hline
\end{tabular}

Teste: Diferença de Proporção

Para ilustrar ainda mais o impacto da ação educativa foram levantados os dados referentes ao faturamento mensal dos curativos no ano de 2015, apresentados na figura 1. Observa-se que no mês de fevereiro foram processados 284 procedimentos, correspondendo ao faturamento do valor de $\mathrm{R} \$$ 9.201,60 (nove mil, duzentos e um reais e sessenta centavos) e em julho, por sua vez, foram processados 776 procedimentos, correspondendo ao valor faturado de $\mathrm{R} \$ 25.142,40$ (vinte e cinco mil, cento e quarenta e dois reais e quarenta centavos).

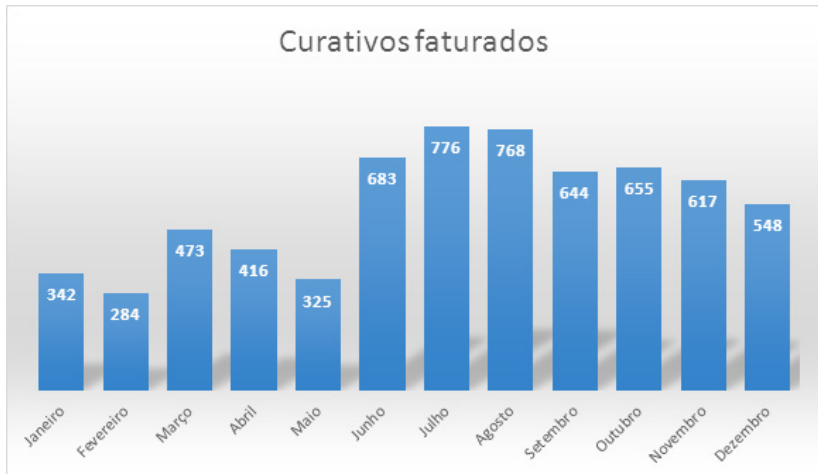

Fonte: CDI 2015.

Figura 1 - Faturamento mensal de curativos no hospital do estudo. Goiânia-GO,Brasil, 2015.
Os valores de faturamento aumentaram significativamente a partir de junho, ou seja, posterior à ação educativa. Após o pico de ascensão da curva apresenta uma tendência de queda, podendo-se inferir a necessidade de ações de educação continuada.

Considerando-se os dois momentos de coleta foram analisadas 532 ocorrências, destas, em 352 $(66,1 \%)$ os curativos foram prescritos; enquanto que em $522(98,1 \%)$ foram executados e devidamente registrados. Ressalta-se que em 170 (32\%) os procedimentos foram realizados sem a devida prescrição correspondente, o que impossibilitou o faturamento. Desta forma, a unidade deixou de faturar o valor de $\mathrm{R} \$ 5.508,00$ (cinco mil, quinhentos e oito reais), levando-se o valor atual de $\mathrm{R} \$ 32,40$ (trinta e dois reais e quarenta centavos) por curativo.

\section{DISCUSSÃO}

Após a atividade de ação educativa, observouse aumento significativo do número dos registros referentes à prescrição do procedimento, checagem e aprazamento, inferindo-se que tais modificações sejam o resultado da ação ocorrida. A atividade desenvolvida com enfermeiros, procurou ressaltar a importância dos registros efetuados sobretudo pela enfermagem, com maior ênfase na prescrição de curativos, devido faturamento envolvido.

A ação educativa é uma ação pontual que possibilita capacitação e ações transformadoras que favorecem mudança de pensamentos e ações. ${ }^{8}$ Entretanto, para que ocorra a mudança comportamental, é necessário o investimento em educação continuada, para desta forma, manter a equipe em constante aperfeiçoamento, ${ }^{9}$ reforçados pelos números encontrados na figura 1.

A educação continuada na enfermagem permite aos profissionais melhoria contínua nos processos de educação e treinamento técnico, desenvolvendo as qualificações de modo a incorporar princípios de humanização, onde a busca pela melhoria assistencial promove o acolhimento ao paciente, sem perder de vista o custo e eficácia do atendimento. ${ }^{10}$

Com o objetivo de promover o conhecimento da equipe, verifica-se a tendência a intenso investimento em educação continuada e permanente. A equipe deve ser constantemente orientada que os registros são a expressão do cuidado produzido e além de refletir a qualidade da assistência, permitem a garantia do pagamento pelos procedimentos realizados. ${ }^{9,11}$ Contudo, apesar da importância da formação continua, em um contexto de escassez de 
recursos, este é o primeiro alvo a ser atingido por cortes no orçamento. ${ }^{12}$ Por esse motivo, reforça-se a importância da sensibilização dos gestores em relação à esta temática.

A prescrição é um importante elo de comunicação escrita entre os diversos profissionais e deve conter elementos mínimos que garantam a eficiência necessária ao processo de assistência, ${ }^{13}$ sendo de total responsabilidade do profissional que a prescreve, sua elaboração deverá ocorrer de forma completa, com informações suficientes para equipe multidisciplinar. ${ }^{14}$ A prescrição do curativo pelo enfermeiro encontra-se devidamente fundamentada pela Resolução n ${ }^{0}$ 358/2009, que dispõe sobre a Sistematização da Assistência de Enfermagem (SAE) e pela Resolução 501/2015, que regulamenta a competência dos enfermeiros no tratamento de feridas. ${ }^{15-16}$

Não se observou mudança significativa na evolução, descrição minuciosa do procedimento realizado ${ }^{17}$ que na dimensão do tratamento de feridas, significa a descrição de diversas características, tais como: o local da lesão, qual o aspecto, como o profissional procedeu, como a classificou, quais recursos utilizou, ou até mesmo como o paciente reagiu ao tratamento. ${ }^{18} \mathrm{~A}$ descrição detalhada do procedimento é de grande importância para a continuidade do cuidado, conferindo qualidade ao atendimento clínico. ${ }^{19}$

O significativo aumento do número de prescrições de curativos realizadas por enfermeiros proporcionalmente em relação àquelas prescritas por médicos reforçam o impacto positivo da ação educativo, visto que os médicos não foram englobados na ação educativa. A elevação no número de registros da execução, efetuados por técnicos e enfermeiros, com proporcional redução dos registros sem identificação refletem maior participação destes profissionais na execução destes cuidados, evidenciando que a equipe passou a valorizar mais o registro desta ação. Estudo corrobora que o enfermeiro vem destacando-se como profissional responsável pelo tratamento de feridas, responsabilizando-se igualmente pelo treinamento de sua equipe para que se desenvolvam ações seguras, sem desperdícios ou retrabalho. ${ }^{20}$

Verificou-se pelo cruzamento dos dados, que alguns procedimentos foram realizados sem que tivessem sido prescritos. Este comportamento, além de ocasionar perdas no faturamento, também pode denotar ênfase do "fazer" em detrimento do "registrar", contribuindo para a invisibilidade da enfermagem. ${ }^{21}$ Estudo conduzido em um hospital privado do Rio de Janeiro identificou que as falhas referentes ao registro relativos à ausência de anotação de enfermagem, resultou em glosas, representando um custo para instituição no valor de $\mathrm{R} \$ 16.085,28 .{ }^{22}$ Nesse contexto, verificou-se a importância do enfermeiro auditor, que deve não somente identificar as falhas nos registros, mas sobretudo, buscar como objetivo principal, a excelência da assistência de enfermagem, não desmerecendo as questões de custos e cobranças hospitalares tradicionalmente contempladas. ${ }^{23}$

A importância da enfermagem em conhecer todo o processo de faturamento e custos do procedimento, é mencionado por estudo que considera o enfermeiro como uma ferramenta de gerenciamento, por desempenhar papel importante para o controle e gestão de recursos físicos, humanos, materiais e financeiros, além de participação em planejamento estratégico. ${ }^{6}$ Já o desconhecimento dos custos pode acarretar análises errôneas sobre os gastos dos setores e, muitas vezes, como consequência, tomadas de decisões inapropriadas às situações existentes. $^{24}$

Desta forma, enfatiza-se que além de investimentos para capacitação conjunta dos profissionais do faturamento e da assistência, o conhecimento sobre os custos dos procedimentos realizados pela enfermagem podem contribuir para melhorar os registros da assistência prestada e diminuir as perdas de faturamento e glosas hospitalares. ${ }^{25}$

\section{CONCLUSÃO}

A ação educativa contribuiu significativamente para o aumento do número de prescrições referentes ao procedimento especial curativo grau II, impactando no aumento do faturamento hospitalar relacionado a este procedimento.

A utilização da estratégia de atividade educativa foi preponderante para a motivação dos profissionais de enfermagem em prescreverem o procedimento, que por vezes era executado sem a devida prescrição correspondente, denotando uma prática que contribui para a invisibilidade da enfermagem.

A tendência dos números do faturamento no decorrer do ano de 2015 demonstram a importância da educação continuada, apontado para a necessidade de que novas ações sejam desenvolvidas, extensivas a todos profissionais envolvidos na assistência e também aos profissionais envolvidos no faturamento.

Espera-se que este estudo possa subsidiar outros, que venham demonstrar a contribuição da 
enfermagem no faturamento hospitalar. Desta forma, recomenda-se que ocorra o esforço em aprimorar os conhecimentos relacionados ao faturamento das ações efetuadas pela enfermagem, assim como o investimento dos gestores em ações de educação continuada.

\section{AGRADECIMENTOS}

À Fundação de Amparo à Pesquisa do Estado de Goiás (FAPEG) e ao Programa de Fortalecimento do SUS.

\section{REFERÊNCIAS}

1. Claudino HG, Gouveia EML, Lopes MEL, Santos SR. Auditoria em registros de enfermagem: revisão integrativa da literatura. Rev Enferm UERJ [Internet]. 2013 cited 2014 Nov 14]; 21(3):397-402. Availeble from: http:/ / www.e-publicacoes.uerj.br/index.php/ enfermagemuerj/article/view/7550/0

2. Mauriz C, Lobo F, Lima RR, Oliveira S, Medeiros G. Faturamanto hospitalar: um passo a mais. Rev Inov Ação. 2012; 1(1):38-44.

3. Silva LCP, Muller DA, Sales PRS, Almeida JL, Barbosa PMK, Melo SMCB. O faturamento hospitalar: quem cuida conhece? RAS. 2013; 15(60):121-6.

4. Cintra FR, Vieira SFA, Hall RJ, Fernandes CR. Information from the invoicing sector as support for decision-making: A case study at the University Hospital of the Federal University of Grande Dourados (UFGD). Rev Cienc Saude Col [Internet]. 2013 [cited 2014 Nov 14]; 18(10):3043-53. Available from: http:/ / www.scielosp.org/pdf/csc/v18n10/ v18n10a29.pdf

5. Bertolini DBB, Meloni DR, Costa FN, Costa AL, Matos PVL. Contrato de gestão: o impacto da contratualização no Centro de Referência da Saúde da Mulher de Ribeirão Preto - MATER. Rev FMRP USP [Internet]. 2015 [2017 Jun 14]; 48(1):19-26. Available from: http://www.revistas.usp.br/rmrp/article/ view/96829

6. Zunta RSB, Castilho V. Billing or nursing procedures at na intensive care unit. Rev Latino-am Enferm [Internet]. 2011 [2017 Jun 14]; 19(3):573-80. Available from: http:// www.scielo.br/scielo.php?script=sci_ar ttext\&pid=S0104-11692011000300017

7. Ministério da Saúde (BR). Sistema de Informação do SUS: Manual Técnico e Operacional do Sistema de Informação Hospitalar. Secretaria de Atenção à Saúde, editor. Secretaria de Atenção à Saúde. Brasília (DF): MS; 2015.

8. Frigo LF, Maurer R, Mattos KM De, Boeira GS, Manfi F, Piaia E, et al. Ação educativa interdisciplinar para pacientes com diabetes na atenção básica: uma revisão bibliográfica. Rev Epidemiol Control Infect. 2012; 2(4):141-3.
9. Silva JS. Audit in health: a new paradigm in the quality of nursing care. Rev Enferm UFPI. 2015 Apr-Jun; 4(2):130-4.

10. Barbosa AP, Eiras FC das, Leão EC, Barnabé AS. Process organization in improvement of health records quality nursing care. Rev Raunp. 2015;7(2):121-30.

11. Salum NC, Prado ML. Continuing education in the development of competences in nurses. Texto Contexto Enferm. 2014 [2017 Jun 14]; 23(2):301-8. Available from: http:/ / www.scielo.br/scielo.php?script=sci_ arttext\&pid=S0104-07072014000200301\&lng=en\&nr $\mathrm{m}=$ iso\&tlng=en

12. Ouellet J, Makamunera J. Avancées en formation infirmière: La formation continue d'infirmières de soins à domicile: retombées perçues au regard de l'amélioration de la qualité des soins aux aînés. Qual Adv Nurs Educ. 2015 [2017 Jun 14]; 1(3). Available from: https://qane-afi.casn.ca/journal/vol1/iss3/6/

13. Pazin-Filho A, Frezza G, Matsuno AK, De Alcantara ST, Cassiolato S, Bitar JPS, et al. Princípios de prescrição médica hospitalar para estudantes de medicina. Rev FMRP USP [Internet]. 2013 [2017 Jun 14]; 46(2):183-94. Available from: http://www. revistas.usp.br/rmrp/article/view/62319

14. Lopes LN, Garcia KP, Dias LG, Soares LR, Leite AM, Silva JAC. Qualidade das prescrições médicas em um Centro de Saúde Escola da Amazônia Brasileira. Rev Soc Bras Clin Med [Internet]. 2014 [2017 Jun 14]; 12(2):1-5. Available from: http://files.bvs.br/ upload/S/1679-1010/2014/v12n2/a4184.pdf

15. Conselho Federal de Enfermagem. Resolução COFEN 358/2009: dispõe sobre a Sistematização da Assistência de Enfermagem [Internet]. Brasília (DF): Cofen; 2009. Available from: http:/ / www.cofen.gov. br/resoluo-cofen-3582009_4384.html

16. Conselho Federal de Enfermagem. Resolução COFEN 501/2015 - Regulamenta a competência da equipe de enfermagem no cuidado às feridas. [Internet]. Brasília (DF): COFEN; 2015 [2017 Jun 14]. Available from: http:/ / www.cofen.gov.br/resolucao-cofenno-05012015_36999.html

17. Barros ALBL, Sanchez CG, Lopes JL, Dell'Acqua MCQ, Lopes MHBM, Silva RCG. Processo de Enfermagem: guia para a prática. Conselho Regional de Enfermagem. São Paulo: COREN-SP; 2015.

18. Santos JB Dos, Porto SG, Suzuki LM, Sostizzo LDRZ, Antoniazzi JL, Echer IC. Avaliação e tratamento de feridas - orientação aos profissionais de saúde. Porto Alegre (RS): UFRGS; 2011.

19. Barbosa SF, Tronchin DMR. Manual for monitoring the quality of nursing home care records. Rev Bras Enferm. 2015 [2017 Jun 14]; 68(2):253-60. Available from: http:/ / www.scielo.br/scielo.php?script=sci_ arttext\&pid=S0034-71672015000200253\&lng=en\&nr $\mathrm{m}=\mathrm{iso} \& \operatorname{lng}=\mathrm{en}$

20. Silva LAP, Shutz V, Machado DA. Partial assessment of the cost of dressings performed at an intensive 
care unit. Rev Enferm UFPE line. 2015 [2017 Jun 14]; 9(3):7031-8. Available from: https:/ / periodicos.ufpe. br/revistas/revistaenfermagem/article/view/10431

21. Rosa LA, Caetano LA, Matos SS, Reis DC. Auditoria como estratégia de avaliação dos registros de enfermagem em unidade de internação pediátrica. REME 2012; 16(1):545-53.

22. Ferreira TS, Souza-Braga AL, Cavalcanti-Valente GS, Souza DF, Carvalho-Alves EM. Auditoria de enfermagem: o impacto das anotações de enfermagem no contexto das glosas hospitalares. Aquichan. 2009; 9(1):38-49.

23. Viana CD, Bragas LZT, Bragas TD, Lazzari DD, Garcia CTF, Moura GMSS. Implementation of concurrent nursing audit: an experience report. Texto Contexto
Enferm [Internet]. 2016 [2017 Jun 14]; 25(1):1-7. Avalible from: htpp://www.scielo.br/pdf/tce/ v25n1/0104-0707-tce25-01-3250014.pdf

24. Menezes JGBRL, Bucchi SM. Auditoria em enfermagem: um instrumento para assistência de qualidade. Rev Enferm UNISA [Internet]. 2011 [cited 2014 Aug 9]; 12(1):68-72. Available from: http:// www.unisa.br/graduacao/biologicas/enfer/revista/ arquivos/2011-1-12.pdf

25. Guerrer GFF, Lima AFC, Castilho V. Study of billing audits in a teaching hospital. Rev Bras Enferm [Internet]. 2015 [2017 Jun 14]; 68(3):414-20. Available from: http:/ / www.scielo.br/scielo.php?script=sci_ arttext\&pid=S0034-71672015000300414\&lng=en\&nr $\mathrm{m}=\mathrm{iso \& t} \operatorname{lng}=\mathrm{en}$ 